\title{
Teaching the Fixed Spinning Top Using Four Alternative Formulations
}

\author{
CHRISTOPHER G. PROVATIDIS \\ School of Mechanical Engineering \\ National Technical University of Athens \\ 9 Iroon Polytechniou, 15780 Zografou \\ GREECE
}

\begin{abstract}
This paper discusses four different approaches that can be followed to derive the equations of motion for a fixed and symmetrical spinning top. Starting from the usual Euler equations in the body-fixed system, after manipulation it is shown that identical equations are derived for the space-fixe system as well. All the three Cartesian components of the angular momentum vector are calculated for both the body- and the space-systems and they are formulated so that they can be used for further numerical analysis. In addition to the classical set, the Euler equations are also easily derived using a rotating system originated at the pivot but not spinning. Moreover, Lagrange equations are derived and the latter are proven to be equivalent with the Euler equations. The best way among these four methods for teaching students is probably the instructor's preference. Moreover, using commercial software, an adequately accurate numerical solution is derived. Not only the position of the spinning top is calculated but also the support forces at the pivot are predicted.
\end{abstract}

Key-Words: - Spinning top, Rigid-body dynamics, Euler equations, Lagrange equations, Runge-Kutta.

Received: October 10, 2020. Revised: March 23, 2021. Accepted: April 13, 2021. Published: April 30, 2021.

\section{Introduction}

The spinning top is the basis for illustrating gyroscopic effects involved in many industrial applications such as inertial sensors for navigation [1], spacecraft attitude control [2], gyroscopic wave energy harvesting [3], and others [4].

First acquaintance with a spinning top occurs in early childhood, when the child watches the unusual behavior of this widely known toy, which seems to be able to defy gravity. In general, the spinning top performs three rotations, which involves precession, nutation and spin.

The mechanics of the spinning top was studied for the first time by Lagrange [5], and after a century by Klein and Sommerfeld [6,7]. In 1897 Klein separately published a shorter monograph [8]. One of the oldest journal papers is probably due to Appel'rot [9]. Relevant books of general interest are $[10,11]$, and Gould [12] includes an extended list of 367 references. Explicit time integration of equations of motion, to give the nutation in terms of elliptic integrals, is cited in the aforementioned books [6,7] as well as in Whittaker [13]. Some useful explanations were given by Zaroodny [14].
Analytical formulas for nutation are reported in some older books [15-18], whereas recent textbooks in physics deal mostly reduce to the regular precession $[19,20]$. A remarkable internet link is [21].

Between several findings regarding spinning tops, in 1889, the Russian mathematician Kovalevskaya showed that the rigid body motion was integrable under certain conditions concerning the ratio $(1: 2)$ of the principal momenta between other parameters; her work was so remarkable that it won her the Bordin prize (1888) [22-25]. Particular reference may be found in the introduction of a book by the French mathematician Michèle Audin [26], who used the term "mysterious (?)" when referring to the Kowalevski case.

The integrability of Euler's equations describing the motion of a spinning top, i.e. the numerical calculation of Euler angles as functions on the elapsed time, has become a matter of intensive research within the last fifteen years. In brief, the numerical solution may sometimes not fulfill the law of energy conservation or may suffer from "gimbal lock" singularities concerning the Euler angles [26-28]. Regarding numerical analysis on the spinning top, an early paper on the numerical integration of the differential equations is probably due to Gorn [29, p.79]. Later, McGill and Long [30] 
studied the case of an unsymmetrical rigid body. Also, Simo and associates have developed numerical schemes to preserve energy and momentum [31,32] and papers therein. Ratiu and Moerbeke [33] have discussed the same matter with main emphasis put on the symplectic structure of the motion. Historical references have been given by Romano [34], whereas some more recent publications are [35-43].

To better understand the necessity of complicated numerical schemes, nine years ago, several simple numerical schemes, such as RungeKutta and Crank-Nicolson, were successfully implemented for two benchmark test cases using inhouse software as well as built-in MATLAB ${ }^{\circledR}$ functions [44]. Therefore, elementary knowledge of Numerical Analysis is sufficient.

During the last decade the research on the spinning top has continued in several directions. For example, particular shapes of spinning tops -for example- one with a spherical bottom end [45], nonlinear phenomena such as the friction at the fixed point [46,47], elastic effects [48], and several types of forced precession effects [49-51] have been reported.

The basic purpose of this paper is to derive the complete nonlinear equations which govern the complex motion of the spinning top consisting of precession, nutation and spin. This is implemented following four alternative formulations with different levels of difficulty in their introduction to students of physics and engineering studies. The equivalency among these formulations is thoroughly commented. Having reported these four approaches and their interrelations in a systematic way, the instructor may be further inspired for specific discussions in the classroom. At the end of the presentation, a benchmark test case is numerically solved in an efficient way. The numerical solution includes the kinematical simulation of the fixed spinning top (i.e., Euler angles) as well as the support forces.

\section{Basic theory}

In general, the position of the spinning top -as a rigid body rotating about a fixed point $\mathrm{O}$ - is determined through three rotational degrees of freedom. The analysis is performed in either the space-fixed $O x_{s} y_{s} z_{s}$ or the body-fixed Cartesian system $O x_{b} y_{b} z_{b}$, with unit vectors $(\vec{i}, \vec{j}, \vec{k})$ and $\left(\vec{i}^{\prime}, \vec{j}^{\prime}, \vec{k}^{\prime}\right)$, respectively.

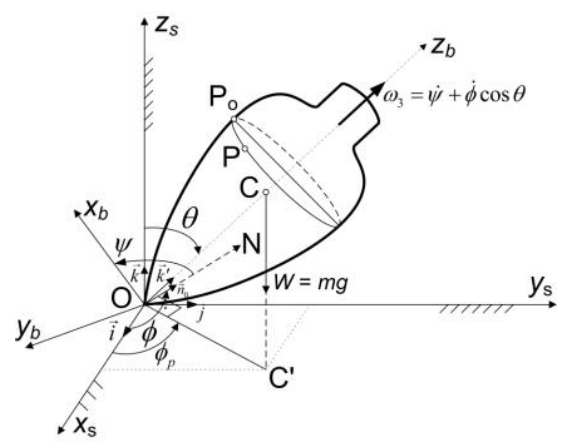

Figure 1: Euler angles of a spinning top.

Let $C$ be the center of mass of the symmetric spinning top, which will be along its long body axis $O z_{b}$.

The normal projection of the aforementioned point $C$ on the horizontal space-fixed plane $O x_{s} y_{s}$ is denoted by $C^{\prime}$, as shown in Fig. 1.

Also, the so-called "line of nodes $O N$ " is defined as the line perpendicular to $\mathrm{OC}^{\prime}$ and lying on the horizontal space-fixed plane $O x_{s} y_{s}$.

The formulation of the celebrated Euler equations is based on the following three Euler angles (see, Fig. 1):

- Azimuthal angle $\phi$ with angular velocity $\omega_{\phi}=\dot{\phi}$, due to rotation about the vertical space $z_{s}$-axis.

- Inclination (lean) angle $\theta$ with angular velocity $\omega_{\theta}=\dot{\theta}$, about the line of nodes $O N$.

- Spin angle $\psi$ with angular velocity $\omega_{\psi}=\dot{\psi}$, about the body-fixed long axis $z_{b}$.

The above details, which are adopted in this paper, were given because different authors may use different sets of angles to describe these orientations, or different names for the same angles, leading to different conventions (see, for example, [52] or [53]).

The instantaneous vector of the angular velocity $\overrightarrow{\boldsymbol{\omega}}$ along the inclined line $O \Omega$ (not shown for avoiding confusion) can be expressed as follows: 


$$
\overrightarrow{\boldsymbol{\omega}}=\omega_{\phi} \vec{k}+\omega_{\theta} \vec{n}_{0}+\omega_{\psi} \vec{k}^{\prime}
$$

where $\vec{k}, \vec{n}_{0}$ and $\vec{k}^{\prime}$ are the unit vectors along the space $z_{s}$-axis, the line of nodes $O N$, and the body $z_{b}$-axis, respectively. In terms of the space unit vectors, the aforementioned unit vector $\vec{n}_{0}$ will be

$$
\vec{n}_{0}=\cos \phi \vec{i}+\sin \phi \vec{j}
$$

while the unit vector along the body-fixed long $z_{b}$ axis

$$
\vec{k}^{\prime}=\sin \theta \cos \phi_{P} \vec{i}+\sin \theta \sin \phi_{P} \vec{j}+\cos \theta \vec{k} .
$$

Since the usual spherical azimuthal angle $\phi_{P}=\square\left(O x_{s}, O C^{\prime}\right)$ (Fig. 1) is related to the Euler angle $\phi$ through the expression

$$
\phi_{P}=\phi-\frac{\pi}{2}
$$

we have $\left(\cos \phi_{P}=\sin \phi\right.$ and $\left.\sin \phi_{P}=-\cos \phi\right)$ thus Eq. (3a) becomes:

$$
\vec{k}^{\prime}=\sin \theta \sin \phi \vec{i}-\sin \theta \cos \phi \vec{j}+\cos \theta \vec{k} .
$$

Therefore, for the space-fixed system we obtain:

$$
\begin{aligned}
\overrightarrow{\boldsymbol{\omega}} & =\left(\omega_{\theta} \cos \phi+\omega_{\psi} \sin \phi \sin \theta\right) \vec{i} \\
& +\left(\omega_{\theta} \sin \phi-\omega_{\psi} \cos \phi \sin \theta\right) \vec{j} \\
& +\left(\omega_{\phi}+\omega_{\psi} \cos \theta\right) \vec{k}
\end{aligned}
$$

Replacing the vector $\overrightarrow{\boldsymbol{\omega}}=\omega_{x} \vec{i}+\omega_{y} \vec{j}+\omega_{z} \vec{k}$ with its associated column-vector $\boldsymbol{\omega}=\left[\begin{array}{lll}\omega_{x} & \omega_{y} & \omega_{z}\end{array}\right]^{T}$, the above expression may be also written in matrix form as follows:

$$
\boldsymbol{\omega} \square\left[\begin{array}{c}
\omega_{x} \\
\omega_{y} \\
\omega_{z}
\end{array}\right]=\left[\begin{array}{l}
0 \\
0 \\
1
\end{array}\right] \omega_{\phi}+\left[\begin{array}{c}
\cos \phi \\
\sin \phi \\
0
\end{array}\right] \omega_{\theta}+\left[\begin{array}{c}
\sin \theta \sin \phi \\
-\sin \theta \cos \phi \\
\cos \theta
\end{array}\right] \omega_{\psi} .
$$

Between several options, the final position $(\phi, \theta, \psi)$ of the spinning top is accomplished by rotating the initial space-fixed system in three successive rotations, as follows [15].

First, the coordinate system is rotated about the space $z_{s}$-axis by the azimuthal angle $\phi$ and this rotation induces a rotation matrix $\mathbf{D}$, i.e. $\xi=\mathbf{D} \mathbf{x}_{s}$, given by

$$
\mathbf{D}=\left[\begin{array}{ccc}
\cos \phi & \sin \phi & 0 \\
-\sin \phi & \cos \phi & 0 \\
0 & 0 & 1
\end{array}\right]
$$

In the abovementioned first rotation, the initial space $x_{s^{-}}$and $y_{s}$-axes take the position $\xi$ (line of nodes) and $\eta$, respectively, both lying on the horizontal space plane $O x_{s} y_{s}$. The third axis, $\zeta$, coincides with the space $z_{s}$-axis. By definition, the $\xi$-axis (line of nodes) is perpendicular to the space $z_{s}$-axis.

Second, the previous system $O \xi \eta \zeta$ is rotated about the abovementioned $\xi$-axis (line of nodes) by the lean angle $\theta$. In this rotation, the abovementioned $\zeta \equiv z$ axis takes the new position $\zeta^{\prime}$. Obviously, the $\xi$-axis (line of nodes) is perpendicular to the body-fixed $z_{s}$-axis. This rotation, $\xi^{\prime}=\mathbf{D} \boldsymbol{\xi}$, is defined by the rotation matrix:

$$
\mathbf{C}=\left[\begin{array}{ccc}
1 & 0 & 0 \\
0 & \cos \theta & \sin \theta \\
0 & -\sin \theta & \cos \theta
\end{array}\right]
$$

Third, the system $O \xi \eta^{\prime} \zeta^{\prime}$ undertakes its last rotation about the $\zeta^{\prime}$ axis. In this rotation, $\mathbf{x}=\mathbf{D} \xi^{\prime}$, the system of the rectangular axes $\xi^{\prime}$ and $\eta^{\prime}$ is rotated by the spin angle $\psi$, thus the rotation matrix is:

$$
\mathbf{B}=\left[\begin{array}{ccc}
\cos \psi & \sin \psi & 0 \\
-\sin \psi & \cos \psi & 0 \\
0 & 0 & 1
\end{array}\right]
$$

The entire rotation is given by the product of all the abovementioned three rotations, in reverse sequence, $\mathbf{x}_{b o d y}=\mathbf{A} \mathbf{x}_{\text {space }}$, as follows:

$\mathbf{A}=\mathbf{B C D}=\left(\begin{array}{ccc}\cos \phi \cos \psi-\sin \phi \cos \theta \sin \psi & \sin \phi \cos \psi+\cos \phi \cos \theta \sin \psi & \sin \theta \sin \psi \\ -\cos \phi \sin \psi-\sin \phi \cos \theta \cos \psi & -\sin \phi \sin \psi+\cos \phi \cos \theta \cos \psi & \sin \theta \cos \psi \\ \sin \phi \sin \theta & -\cos \phi \sin \theta & \cos \theta\end{array}\right)$

Note that since the line of nodes $O N$ is perpendicular to both $O z_{s}$ and $O z_{b}$ axes, we have 
$\vec{n}_{0}=\left(\vec{k} \times \vec{k}^{\prime}\right) /\left|\vec{k} \times \vec{k}^{\prime}\right|$, and it lies on the horizontal plane $O x_{s} y_{s}$.

Based on the above transformation matrices, we can derive the body components of each component $\left(\omega_{\phi}, \omega_{\theta}, \omega_{\psi}\right)$ in a different way. In more details, the quantities $\omega_{\phi}, \omega_{\theta}$ and $\omega_{\psi}$ are not rotated in the same manner, but each of them utilizes one among the three abovementioned matrices, as follows:

$$
\begin{aligned}
& \left(\boldsymbol{\omega}_{\phi}\right)_{b o d y}=\mathbf{A} \omega_{\phi}, \\
& \left(\boldsymbol{\omega}_{\theta}\right)_{b o d y}=\mathbf{B} \omega_{\theta}, \\
& \left(\boldsymbol{\omega}_{\psi}\right)_{b o d y}=\left[\begin{array}{lll}
0 & 0 & \omega_{\psi}
\end{array}\right]^{T}
\end{aligned}
$$

Therefore, the three Cartesian components of the body-fixed angular velocity are:

$$
\boldsymbol{\omega}_{b o d y}=\left[\begin{array}{c}
\omega_{1} \\
\omega_{2} \\
\omega_{3}
\end{array}\right]=\mathbf{A}\left[\begin{array}{c}
0 \\
0 \\
\omega_{\phi}
\end{array}\right]+\mathbf{B}\left[\begin{array}{c}
\omega_{\theta} \\
0 \\
0
\end{array}\right]+\left[\begin{array}{c}
0 \\
0 \\
\omega_{\psi}
\end{array}\right]
$$

Substituting (8) and (9) into (11), one obtains the well-known formulas, in which the subscripts $(1,2,3)$ refer to principal body axes:

$$
\begin{aligned}
& \omega_{1}=\dot{\phi} \sin \theta \sin \psi+\dot{\theta} \cos \psi, \\
& \omega_{2}=\dot{\phi} \sin \theta \cos \psi-\dot{\theta} \sin \psi, \\
& \omega_{3}=\dot{\phi} \cos \theta+\dot{\psi} .
\end{aligned}
$$

Although we have previously derived the expression of the space angular velocity $\boldsymbol{\omega} \square \boldsymbol{\omega}_{\text {space }}$ (see, Eqs.

(4) and (5)), here we can see an alternative way based on matrix transformation. Actually, inverting the relation $\boldsymbol{\omega}_{b o d y}=\mathbf{A} \boldsymbol{\omega}_{\text {space }}$, and considering that $\mathbf{A}^{-1}=\mathbf{A}^{T}$, we receive $\boldsymbol{\omega}_{\text {space }}=\mathbf{A}^{T} \boldsymbol{\omega}_{\text {body }}$, which is eventually written as:

$$
\begin{aligned}
\boldsymbol{\omega}_{\text {space }} & =\left[\begin{array}{c}
\omega_{x} \\
\omega_{y} \\
\omega_{z}
\end{array}\right]=\left[\begin{array}{l}
0 \\
0 \\
1
\end{array}\right] \omega_{\phi}+\left[\begin{array}{c}
\cos \phi \\
\sin \phi \\
0
\end{array}\right] \omega_{\theta} \\
& +\left[\begin{array}{c}
\sin \theta \sin \phi \\
-\sin \theta \cos \phi \\
\cos \theta
\end{array}\right] \omega_{\psi}
\end{aligned}
$$

Obviously, the two procedures are equivalent, thus Eq. (5) and (13) are identical, as anticipated.

\section{Equations in the body-fixed system}

The first approach of this paper is to apply the full Euler equations. These equations implement Newton's second law in rotation. From classical textbooks such as [16], it is well known that Euler equations can be written (in the body-fixed system) in the form of three equations:

$$
\begin{aligned}
& I_{1} \dot{\omega}_{1}-\left(I_{2}-I_{3}\right) \omega_{2} \omega_{3}=M_{1}, \\
& I_{2} \dot{\omega}_{2}-\left(I_{3}-I_{1}\right) \omega_{3} \omega_{1}=M_{2}, \\
& I_{3} \dot{\omega}_{3}-\left(I_{1}-I_{2}\right) \omega_{1} \omega_{2}=M_{3} .
\end{aligned}
$$

Although vector analysis is applicable as done in [16], we provide an alternative proof in Appendix $A$, which is based on matrix transformation along with tensors.

In case that the moments $\left(M_{1}, M_{2}, M_{3}\right)$ vanish or they are given functions of $\left(\omega_{1}, \omega_{2}, \omega_{3}\right)$, we can put the system of Eqs. (14) in the classical form [41,42]:

$$
\begin{aligned}
& \dot{\omega}_{1}-\frac{\left(I_{2}-I_{3}\right)}{I_{1}} \omega_{2} \omega_{3}=\frac{M_{1}}{I_{1}} \\
& \dot{\omega}_{2}-\frac{\left(I_{3}-I_{1}\right)}{I_{2}} \omega_{3} \omega_{1}=\frac{M_{2}}{I_{2}} \\
& \dot{\omega}_{3}-\frac{\left(I_{1}-I_{2}\right)}{I_{3}} \omega_{1} \omega_{2}=\frac{M_{3}}{I_{3}}
\end{aligned}
$$

to which a Runge-Kutta method is applicable. Otherwise, we have to follow a different way, dealing with the original quantities $(\phi, \theta, \psi)$. And this is the beginning of our central discussion of this paper, which will be reduced to a symmetrical top for which $I_{1}=I_{2}$, Note that since the support is frictionless, we have $M_{3}=0$.

Since $I_{1}=I_{2}$ and $M_{3}=0$, Eq. (15c) yields $\dot{\omega}_{3}=0$, that is $\omega_{3}=$ const. Moreover, if the spinning top operates only due to its weight thus leading to the space torque $\mathbf{M}_{\text {space }}=m g l \sin \theta\left[\begin{array}{lll}\cos \phi & \sin \phi & 0\end{array}\right]^{T}$ (for explanations, see Appendix $B$ ), after manipulations the transformation $\mathbf{M}_{\text {body }}=\mathbf{A} \mathbf{M}_{\text {space }}$ yields the body moments $M_{1}=m g l \sin \theta \cos \psi$ and 
also $M_{2}=-m g l \sin \theta \sin \psi$, thus the entire bodyfixed moment vector becomes:

$$
\left\{\begin{array}{l}
M_{1} \\
M_{2} \\
M_{3}
\end{array}\right\}=m g l \sin \theta\left\{\begin{array}{c}
\cos \psi \\
-\sin \psi \\
0
\end{array}\right\}
$$

Substituting Eq.(12) into Eq.(14abc), after manipulation it will be found that Eqs. (14a) and (14b) can be written together in the following matrix form:

$$
\left(\left[\begin{array}{c:c}
d & e \\
\hdashline-e & d
\end{array}\right]-m g l \sin \theta\left[\begin{array}{cc}
0 & 1 \\
-1 & 0
\end{array}\right]\right) \cdot\left[\begin{array}{l}
\sin \psi \\
\cos \psi
\end{array}\right]=\left[\begin{array}{l}
0 \\
0
\end{array}\right]
$$

where

$$
d=I_{1} \ddot{\phi} \sin \theta+2 I_{1} \dot{\phi} \dot{\theta} \cos \theta-I_{3} \omega_{3} \dot{\theta}
$$

and

$$
e=I_{1} \ddot{\theta}-I_{1} \dot{\phi}^{2} \sin \theta \cos \theta+I_{3} \omega_{3} \dot{\phi} \sin \theta
$$

Dropping out the factor $\left[\begin{array}{ll}\sin \psi & \cos \psi\end{array}\right]^{T}$ in Eq.(17), we have $d=0$ and $e=m g l \sin \theta$. Therefore, the two governing equations will be:

$$
I_{1} \ddot{\phi} \sin \theta+2 I_{1} \dot{\phi} \dot{\theta} \cos \theta-I_{3} \omega_{3} \dot{\theta}=0
$$

and

$$
I_{1} \ddot{\theta}-I_{1} \dot{\phi}^{2} \sin \theta \cos \theta+I_{3} \omega_{3} \dot{\phi} \sin \theta=m g l \sin \theta
$$

Later we shall see that the first Euler equation [i.e. Eq. (18b)], also written in the form $\left(I_{1} \dot{\phi} \sin ^{2} \theta+I_{3} \omega_{3} \cos \theta\right)^{\square}=0$, dictates that the component of the angular momentum in the space $z_{s}$-axis is a constant (first invariant). Therefore, Eq. (18b) is practically an ODE of the first order in $\phi$, whereas the second Euler equation [i.e. Eq. (19b)] is an ODE of the second order in $\theta$.

Remark: In the body-fixed system, the components of the derivatives of the angular momentum are $\left(I_{1} \dot{\omega}_{1}, I_{2} \dot{\omega}_{2}, I_{3} \dot{\omega}_{3}\right)$, while the external moments are $\left(M_{1}, M_{2}, M_{3}\right)$. One may observe in Eqs. (14abc) that there is a difference between these two quantities. In other words, the Newton's second law is not applicable to the bodyfixed system 'as is'.

\section{Equations in the space-fixed system}

Let us now move to the second approach of this paper. This section has been slightly influenced by a recent work [54], which however was concerned with the free fall and not the fixed spinning top of this paper.

In the body fixed system, the vector of the angular momentum is

$$
\mathbf{L}_{\text {body }}=\left\lfloor\begin{array}{c}
I_{1} \omega_{1} \\
I_{2} \omega_{2} \\
I_{3} \omega_{3}
\end{array}\right\rfloor
$$

Based on the well known transformation of first order tensors, the same vector in the space system will be

$\mathbf{L}_{\text {space }}=\mathbf{A}^{T} \mathbf{L}_{\text {body }}=\left(\begin{array}{ccc}\cos \phi \cos \psi-\sin \phi \cos \theta \sin \psi & -\cos \phi \sin \psi-\sin \phi \cos \theta \cos \psi & \sin \phi \sin \theta \\ \sin \phi \cos \psi+\cos \phi \cos \theta \sin \psi & -\sin \phi \sin \psi+\cos \phi \cos \theta \cos \psi & -\cos \phi \sin \theta \\ \sin \theta \sin \psi & \sin \theta \cos \psi & \cos \theta\end{array}\right)\left[\begin{array}{c}I_{1} \omega_{1} \\ I_{2} \omega_{2} \\ I_{3} \omega_{3}\end{array}\right]$

For the symmetrical spinning top (i.e. with $I_{1}=I_{2}$ ), substituting Eq. (12) into Eq. (21), after manipulation the latter yields the components of the vector of the space momentum as follows:

$$
\mathbf{L}_{\text {space }}=\left[\begin{array}{lll}
L_{x} & L_{y} & L_{z}
\end{array}\right]^{T}
$$

with:

$$
\begin{aligned}
& L_{x}=I_{1}(\dot{\theta} \cos \phi-\dot{\phi} \cos \theta \sin \theta \sin \phi)+I_{3} \omega_{3} \sin \phi \sin \theta \\
& L_{y}=I_{1}(\dot{\theta} \sin \phi+\dot{\phi} \cos \theta \sin \theta \cos \phi)-I_{3} \omega_{3} \cos \phi \sin \theta \\
& L_{z}=I_{1} \dot{\phi} \sin ^{2} \theta+I_{3} \omega_{3} \cos \theta
\end{aligned}
$$

Taking the first derivatives of the above expressions (angular momentum components) with respect to the time $t$, and equating them with the external torques $\mathbf{M}_{\text {space }}$ due to the weight of the spinning top (Newton's second law in rotation), i.e. $\mathbf{M}_{\text {space }}=m g l \sin \theta\left[\begin{array}{lll}\cos \phi & \sin \phi & 0\end{array}\right]^{T}$, we have:

$$
\begin{aligned}
\dot{L}_{x} & =I_{1}\left(\ddot{\theta} \cos \phi-\ddot{\phi} \sin \theta \cos \theta \sin \phi-\dot{\phi}^{2} \sin \theta \cos \theta \cos \phi-2 \dot{\phi} \dot{\theta} \sin \phi \cos ^{2} \theta\right) \\
& +I_{3} \omega_{3}(\dot{\phi} \sin \theta \cos \phi+\dot{\theta} \sin \phi \cos \theta) \\
& =m g l \sin \theta \cos \phi
\end{aligned}
$$


$\dot{L}_{y}=I_{1}\left(\ddot{\theta} \sin \phi+\ddot{\phi} \sin \theta \cos \theta \cos \phi-\dot{\phi}^{2} \sin \theta \cos \theta \sin \phi+2 \dot{\phi} \dot{\theta} \cos \phi \cos ^{2}(24 \mathrm{~b})\right.$

$-I_{3} \omega_{3}(-\dot{\phi} \sin \theta \sin \phi+\dot{\theta} \cos \phi \cos \theta)$

$=m g l \sin \theta \sin \phi$

$$
\dot{L}_{z}=\left(I_{1} \dot{\phi} \sin ^{2} \theta+I_{3} \omega_{3} \cos \theta\right)^{\sqcup}=0
$$

In order to simplify the above expressions, we first start with the last of them, i.e. with Eq. (24c), the easiest. Actually, performing the derivative of the term $\left(I_{1} \dot{\phi} \sin ^{2} \theta+I_{3} \omega_{3} \cos \theta\right)$ and equating to zero, we obtain

$$
\dot{L}_{z}=\left(I_{1} \ddot{\phi} \sin \theta+2 I_{1} \dot{\phi} \dot{\theta} \cos \theta-I_{3} \omega_{3} \dot{\theta}\right) \sin \theta=0,
$$

thus dropping out the $\sin \theta$, we eventually obtain:

$$
I_{1}(\ddot{\phi} \sin \theta+2 \dot{\phi} \dot{\theta} \cos \theta)-I_{3} \omega_{3} \dot{\theta}=0
$$

One may observe that Eq. (25) is identical with Eq. (18b), which was previously derived from the body system considerations.

Now we continue with the term $\dot{L}_{x}$ (Eq. (24a)], which is written as follows:

$$
\begin{aligned}
\dot{L}_{x}= & {\left[-I_{1}(\ddot{\phi} \sin \theta+2 \dot{\phi} \dot{\theta} \cos \theta)+I_{3} \omega_{3} \dot{\theta}\right] \sin \phi \cos \theta } \\
& +I_{1}\left(\ddot{\theta} \cos \phi-\dot{\phi}^{2} \sin \theta \cos \theta \cos \phi\right)+I_{3} \omega_{3}(\dot{\phi} \sin \theta \cos \phi) \\
& =m g l \sin \theta \cos \phi
\end{aligned}
$$

By virtue of Eq. (25), the term into the bracket [...] of Eq. (26) vanishes, and after dropping out the common factor $\cos \phi$, the final ODE becomes:

$$
I_{1}\left(\ddot{\theta}-\dot{\phi}^{2} \sin \theta \cos \theta\right)+I_{3} \omega_{3} \dot{\phi} \sin \theta=m g l \sin \theta
$$

One may observe that Eq. (27) is identical with Eq. (19b), which was previously derived from the body system considerations.

It is easy to see that both the $x$ - and $y$ components, ( $\dot{L}_{x}$ in Eq. (24a), and $\dot{L}_{y}$ in Eq. (24b)), include the second derivatives, $\ddot{\phi}$ and $\ddot{\theta}$. They also have identical terms in $\theta$, whereas regarding the $\phi$-variable the $\cos \phi$ of the first is replaced by $\sin \phi$ of the second, and $\sin \phi$ of the first is replaced by $(-\cos \phi)$ of the second one. This remark implies that the $x$-component is merely rotated by 90 degrees, thus these two equations are linearly dependent and it is anticipated to give the same result.
Actually, by arranging the terms of $\dot{L}_{y}$ we obtain:

$$
\begin{aligned}
\dot{L}_{y} & =\left[I_{1}(\ddot{\phi} \sin \theta+2 \dot{\phi} \dot{\theta} \cos \theta)-I_{3} \omega_{3} \dot{\theta}\right] \cos \phi \cos \theta \\
& +I_{1}\left(\ddot{\theta} \sin \phi-\dot{\phi}^{2} \sin \theta \cos \theta \sin \phi\right)+I_{3} \omega_{3}(\dot{\phi} \sin \theta \sin \phi) \\
& =m g l \sin \theta \sin \phi
\end{aligned}
$$

As previously happened for $\dot{L}_{x}$, the term in the brackets of Eq. (28) vanishes, and after dropping out the common factor $\sin \phi$, we derive again Eq. (27).

In other words, the three constitutive equations of motion, i.e. $\dot{L}_{x}=m g l \sin \theta \cos \phi$, $\dot{L}_{y}=m g l \sin \theta \sin \phi$ and $\dot{L}_{z}=0$ give a second order system of only two ODEs in $\phi$ and $\theta$. We recall that, as discussed at the end of section 3 , the first of them is practically of the first order. The solution of this system fully determines the visual position of the flywheel in spherical coordinates but does not include the spin angle $\psi$, if it is desired. The latter can be easily calculated from the condition $\omega_{3}=\dot{\phi} \cos \theta+\dot{\psi}=$ const., first by calculating to derivative $\dot{\psi}$ and then by integration. Again, if we need to calculate the function $\psi(t)$, then we have to include the equation $\dot{\psi}=\omega_{3}-\dot{\phi} \cos \theta$ into the equations system for the Runge-Kutta or any relevant time integration method.

\section{A third approach}

Both of the two abovementioned approaches, i.e. the body-fixed and particularly the space fixed systems, impose significant complexity and may terrify the student. This approach is inspired by the fact that, in both the previous formulations (i.e. in the body and space systems), the spin term was eventually suppressed. In simple cases such as the regular precession, classical books on tensor analysis [53], have followed this way.

In more details, we consider a particular body fixed system of which the $z b$-axis (with unit vector $\mathbf{i}_{3}$ along OC, where $\mathrm{O}$ is pivot and $\mathrm{C}$ is the center of mass) always coincide with the long axis of the spinning top but does not rotate in the spin direction. While Hay [53] chooses the unit vector $\mathbf{i}_{1}$ so as to be directed upwards, for reasons of plurality, and in 
order to be consistent with previous literature [49], we consider it in the opposite direction, as shown in Fig. 2.

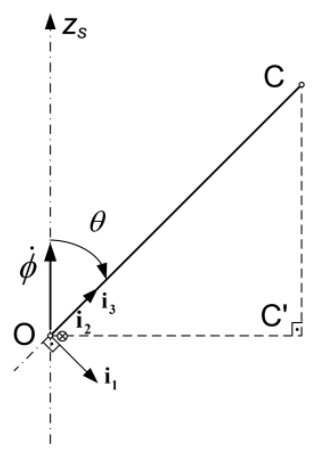

Figure 2: Definition of the rotating system $\left(\mathbf{i}_{1}, \mathbf{i}_{2}, \mathbf{i}_{3}\right)$ at the pivot $\mathrm{O}$.

Both the (i $\mathbf{i}_{1}$, and $\mathbf{i}_{3}$ ) lie on the plane (PCC'), where $\mathrm{C}^{\prime}$ is the projection of $\mathrm{C}$ on the horizontal plane $O x_{s} y_{s}$.

In the case of the fixed top, the center of moments is taken at the pivot $\mathrm{O}$ (axis origin), so as the moments of the reaction forces vanish. Alternatively, one could take the moments with respect to the center of mass but then one has to write the equations of motion for the translational motion as well. The latter approach is more general and has been previously used for the forced precession [49], but not for the fixed top.

Dealing with the vector of the angular momentum $\mathbf{L}$ in an analogous way as the position vector $\overrightarrow{\mathbf{r}}$ (where the total velocity equals to the sum of the relative velocity plus the cross product $\overrightarrow{\boldsymbol{\Omega}} \times \overrightarrow{\mathbf{r}}$ ), the Newton's second law for the rotational motion becomes (see, [16]):

$$
\overrightarrow{\boldsymbol{\tau}}_{e x t}=\frac{d \overrightarrow{\mathbf{L}}_{\text {total }}}{d t}=\left(\frac{d \overrightarrow{\mathbf{L}}}{d t}\right)_{\text {rel }}+\overrightarrow{\mathbf{\Omega}} \times \overrightarrow{\mathbf{L}}
$$

Concerning (29), Fig. 2 depicts that the vector of the angular velocity is given by:

$$
\boldsymbol{\Omega}=\left[\begin{array}{lll}
-\dot{\phi} \sin \theta & \dot{\theta} & \dot{\phi} \cos \theta
\end{array}\right]^{T},
$$

while the angular momentum is given in terms of the momenta of inertia $\left(I_{1}, I_{2}, I_{3}\right)$ with respect to the fixed pivot $\mathrm{O}$ as follows:

$$
\mathbf{L}=\left[\begin{array}{lll}
-I_{1} \dot{\phi} \sin \theta & I_{2} \dot{\theta} & I_{3}(\dot{\phi} \cos \theta+\dot{\psi})
\end{array}\right]^{T}
$$

In (30) and (31), it holds that:

$$
\phi=\phi_{P}+\frac{\pi}{2}
$$

Substituting (29) and (30) into (28), and since the symmetry dictates that $I_{1}=I_{2}$, one obtains:

$$
\frac{d \overrightarrow{\mathbf{L}}_{\text {total }}}{d t}=\left[\begin{array}{c}
\dot{L}_{x} \\
\dot{L}_{y} \\
\dot{L}_{z}
\end{array}\right]=\left[\begin{array}{c}
I_{1}(-\ddot{\phi} \sin \theta-2 \dot{\phi} \dot{\theta} \cos \theta)+I_{3} \dot{\theta}(\dot{\phi} \cos \theta+\dot{\psi}) \\
-I_{1}\left(-\ddot{\theta}+\dot{\phi}^{2} \sin \theta \cos \theta\right)+I_{3} \dot{\phi} \sin \theta(\dot{\phi} \cos \theta+\dot{\psi}) \\
I_{3}(\dot{\phi} \cos \theta+\dot{\psi})
\end{array}\right]
$$

Setting $\dot{\phi} \cos \theta+\dot{\psi}=\omega_{3}$, and considering that the external moment in the space-fixed system is given by (see, Appendix B):

$$
\boldsymbol{\tau}_{e x t}=m g l \sin \theta\left[\begin{array}{c}
\cos \phi \\
\sin \phi \\
0
\end{array}\right]
$$

the first Euler equation in Eq. (33) is identical with the Eq. (25) and (18b), the second Euler equation is identical with Eq. (27) and (19b), whereas the third one depicts that $\omega_{3}$ is a constant (invariant).

\section{Lagrangian approach}

This section is the fourth approach of this paper.

The implementation of Lagrange's equations is a standard procedure which is preferred in complicated cases because it avoids the free-body diagrams and the associated application of Newton's second law to each of them. In our case of the fixed spinning top, the Lagrangian is given by (see, $[16,17])$ :

$$
\tilde{L}=T-V=\frac{1}{2}\left(I_{1} \omega_{1}^{2}+I_{2} \omega_{2}^{2}+I_{3} \omega_{3}^{2}\right)-m g l \cos \theta
$$

Substituting (12) into (35) and considering again a symmetrical top, i.e. $I_{1}=I_{2}$, the latter takes the form:

$$
\tilde{L}=\frac{I_{1}}{2}\left(\dot{\theta}^{2}+\dot{\phi}^{2} \sin ^{2} \theta\right)+\frac{I_{3}}{2} \omega_{3}^{2}-m g l \cos \theta
$$

The Lagrange's equations are written as follows:

$$
\frac{d}{d t}\left(\frac{\partial \tilde{L}}{\partial \dot{q}}\right)-\frac{\partial \tilde{L}}{\partial q}=M_{q}
$$

Substituting (36) into (37) and assuming that the only load is due to the weight $W=m g$ of the spinning top, the right-hand sides $\left(M_{\phi}, M_{\theta}, M_{\psi}\right)$ of Eq. correspond to the moments $M_{z_{s}}=0$, 
$M_{O N}=m g l \sin \theta(O N=$ line of nodes $)$ and $M_{z_{b}}=0$, respectively. As a result, Eq. (37) becomes:

$$
\frac{d}{d t}\left(I_{1} \dot{\phi} \sin ^{2} \theta+I_{3} \omega_{3} \cos \theta\right)=0,
$$

$I_{1} \ddot{\theta}-I_{1} \dot{\phi}^{2} \sin \theta \cos \theta+I_{3} \omega_{3} \dot{\phi} \sin \theta=m g l \sin \theta$

$$
I_{3} \frac{d \omega_{3}}{d t}=0
$$

One may observe that Eq. (38a) and Eq. (38b) do not include the Euler angle $\psi$. Again, the first Euler equation (38a) is identical with Eq. (25) and (18b), whereas the second one is identical with either of (27) and (19b).

In this procedure, again Eq. (38a) dictates that the value of the component of the angular momentum towards the space $z_{s}$-axis is a constant (first invariant of the system), i.e.

$$
p_{\phi}=\partial L / \partial \dot{\phi}=I_{1} \dot{\phi} \sin ^{2} \theta+I_{3} \omega_{3} \cos \theta=c_{1},
$$

where the constant value $c_{1}$ in (39a) is directly determined in terms of three initial conditions $\left(\dot{\phi}_{0}, \theta_{0}, \dot{\psi}_{0}\right)$, as follows:

$$
c_{1}=I_{1} \dot{\phi}_{0} \sin ^{2} \theta_{0}+I_{3}\left(\dot{\phi}_{0} \cos \theta_{0}+\dot{\psi}_{0}\right) \cos \theta_{0} .
$$

Equation (38c) depicts that $\omega_{3}$ is a constant, which can be calculated in terms of three initial conditions $\left(\dot{\phi}_{0}, \theta_{0}, \dot{\psi}_{0}\right)$ by

$$
\omega_{3}=\dot{\phi}_{0} \cos \theta_{0}+\dot{\psi}_{0}
$$

Since $\omega_{3}$ is a constant, the second invariant of the system (angular momentum towards the body $z_{b}$ axis) is:

$$
p_{\psi}=\frac{\partial L}{\partial \dot{\psi}}=I_{3} \omega_{3}
$$

Usually, a criterion to test the accuracy of the numerical integration is the energy conservation, which is given by the form $(E=T+V)$ as follows:

$$
E=\frac{1}{2} I_{1}\left(\dot{\theta}^{2}+\dot{\phi}^{2} \sin ^{2} \theta\right)+\frac{1}{2} I_{3} \omega_{3}^{2}+m g l \cos \theta=c_{2}
$$

As previously, the value $c_{2}$ in (42) is directly determined in terms of the initial conditions and particularly it requires four of them, i.e. $\left(\dot{\phi}_{0}, \theta_{0}, \dot{\theta}_{0}, \dot{\psi}_{0}\right)$.

\section{Decoupled equation for the lean angle}

This idea was presented in previous works of forced precession [49,51] or free-fall [54], but is also applicable to the fixed top [44] which is studied in this paper. In more detail, Eq. (24c) (i.e. the first of Euler equations) dictates that the quantity $I_{1} \dot{\phi} \sin ^{2} \theta+I_{3} \omega_{3} \cos \theta$ is the constant $c_{1}$, which is easily calculated in terms of the initial conditions $\left(\dot{\phi}_{0}, \theta_{0}\right)$, i.e., $\quad c_{1}=I_{1} \dot{\phi}_{0} \sin ^{2} \theta_{0}+I_{3} \omega_{3} \cos \theta_{0}$, as previously was found in Eq. (39b) and Eq. (40).

Solving Eq. (39a) in $\dot{\phi}$ we receive:

$$
\dot{\phi}=\frac{\left(c_{1}-I_{3} \omega_{3} \cos \theta\right)}{I_{1} \sin ^{2} \theta}
$$

Then, substituting Eq. (43) into Eq. (27), one eventually obtains the following single nonlinear ODE:

$$
\begin{aligned}
\ddot{\theta} & =\frac{m g l \sin \theta}{I_{1}} \\
& -\frac{\left(I_{3} \omega_{3}-c_{1} \cos \theta\right)\left(c_{1}-I_{3} \omega_{3} \cos \theta\right)}{I_{1}^{2} \sin ^{3} \theta}
\end{aligned}
$$

In other words, the lean angle $\theta(t)$ fulfils an ordinary differential equation of second order, which is quite independent.

Having said this, we should make clear that the first of the initial conditions $\left(\theta_{0}, \dot{\theta}_{0}\right)$ depends on all the three Euler angles, i.e. particularly on the quantities $\left(\dot{\phi}_{0}, \theta_{0}, \dot{\psi}_{0}\right)$ which form the constant $c_{1}$ [see, Eq. (39b)], while the second one $\left(\dot{\theta}_{0}\right)$ is an independent quantity which affects the motion of the spinning top.

\section{Support forces}

\subsection{Cartesian system}

As the spinning top moves, in addition to the weight $W=m g$, an inclined reaction force $\vec{R}$ with Cartesian components $\left(R_{x}, R_{y}, R_{z}\right)$ is exerted from the ground to the spinning top at the support point $O$. Based on Newton's second law for translation, 


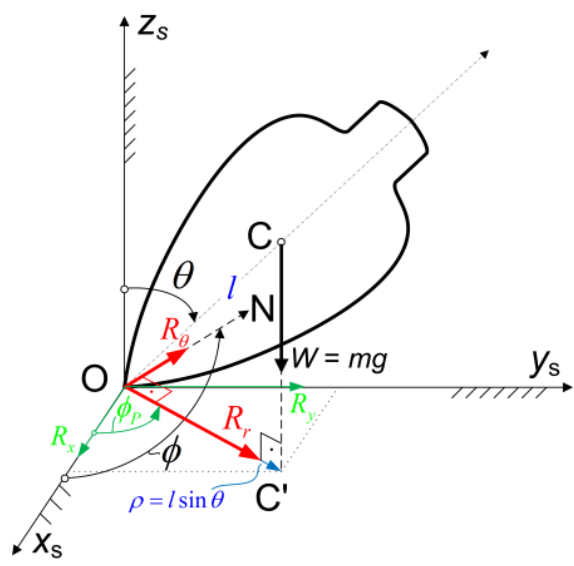

Figure 3: Cartesian and polar force components.

$$
\begin{aligned}
& \sum F_{x}=m \ddot{x}_{c} \\
& \sum F_{y}=m \ddot{y}_{c} \\
& \sum F_{z}=m \ddot{z}_{c},
\end{aligned}
$$

the aforementioned support forces are calculated in terms of the acceleration $\left(\ddot{x}_{c}, \ddot{y}_{c}, \ddot{z}_{c}\right)$ at the center of mass $C$, as follows:

$$
\begin{aligned}
& R_{x}=m \ddot{x}_{c} \\
& R_{y}=m \ddot{y}_{c} \\
& R_{z}=m\left(\ddot{z}_{c}+g\right) .
\end{aligned}
$$

Moreover, the Cartesian coordinates of the center of mass $C$ are given in terms of the radial distance $\rho$ $\left(=O C^{\prime}=l \sin \theta\right)$ on the horizontal plane $O x y$, the polar angle $\phi_{p}$ and the radial distance $l$ of the center of mass $C$ from the fixed point $O$, by $x_{c}=\rho \cos \phi_{p}, y_{c}=\rho \sin \phi_{p}, z_{c}=l \cos \theta$ (Fig. 1).

However, due to Eq. (3b), we have:

$$
\cos \phi_{p}=\sin \phi \quad \text { and } \quad \sin \phi_{p}=-\cos \phi .
$$

Therefore, eventually we have:

$$
\begin{aligned}
& x_{c}=l \sin \phi \sin \theta, \\
& y_{c}=-l \cos \phi \sin \theta, \\
& z_{c}=l \cos \theta
\end{aligned}
$$

Taking the second derivatives of Eqs. (48), and then substituting into Eq. (46), the latter becomes:

$$
\begin{aligned}
R_{x} & =m l[\ddot{\phi} \cos \phi \sin \theta+\ddot{\theta} \sin \phi \cos \theta \\
& \left.-\left(\dot{\phi}^{2}+\dot{\theta}^{2}\right) \sin \phi \sin \theta+2 \dot{\phi} \dot{\theta} \cos \phi \cos \theta\right]
\end{aligned}
$$

$$
\begin{aligned}
R_{y} & =m l[\ddot{\phi} \sin \phi \sin \theta-\ddot{\theta} \cos \phi \cos \theta \\
& \left.+\left(\dot{\phi}^{2}+\dot{\theta}^{2}\right) \cos \phi \sin \theta+2 \dot{\phi} \dot{\theta} \sin \phi \cos \theta\right] \\
R_{z} & =m\left[g-l\left(\ddot{\theta} \sin \theta+\dot{\theta}^{2} \cos \theta\right)\right]
\end{aligned}
$$

\subsection{Polar system}

When the reference system $O x y$ rotates by the angle $\phi_{p}$ the transformation matrix is given by:

$$
\mathbf{T}=\left(\begin{array}{cc}
\cos \phi_{p} & \sin \phi_{p} \\
-\sin \phi_{p} & \cos \phi_{p}
\end{array}\right),
$$

which due to Eq. (43) becomes:

$$
\mathbf{T}=\left(\begin{array}{cc}
\sin \phi & -\cos \phi \\
\cos \phi & \sin \phi
\end{array}\right),
$$

If now the subscript $r$ denotes the direction of the radius $\rho$ measured from the axis origin $O$ to the projection $C^{\prime}$ (i.e. by rotating the axis $O x$ from its original space position to the final position $O C^{\prime}$, we have:

$$
\left\lfloor\begin{array}{l}
R_{r} \\
R_{\theta}
\end{array}\right\rfloor=\mathbf{T}\left\lfloor\begin{array}{l}
R_{x} \\
R_{y}
\end{array}\right\rfloor
$$

Substituting Eq. (50b) into Eq. (51) we receive:

$$
\begin{aligned}
& R_{r}=R_{x} \sin \phi-R_{y} \cos \phi \\
& R_{\theta}=R_{x} \cos \phi+R_{y} \sin \phi
\end{aligned}
$$

Substituting Eqs. (49a,b) into Eq. (52), we obtain:

$$
\begin{aligned}
& R_{r}=m l\left[\ddot{\theta} \cos \theta-\left(\dot{\phi}^{2}+\dot{\theta}^{2}\right) \sin \theta\right] \\
& R_{\theta}=m l[\ddot{\phi} \sin \theta+2 \dot{\phi} \dot{\theta} \cos \theta]
\end{aligned}
$$

Therefore, the vertical support force is given by Eq. (49c), while the horizontal force components are given either in Cartesian form [Eqs. $(49 a, b)]$ or in polar form [Eqs. $(53 a, b)]$. One may observe that the component $R_{\theta}$ is directed towards the line of points ON.

\section{Numerical solution and application example}

There are several ways to obtain the numerical solution, of which detailed discussion is out the scope of this paper. Some of them have been previously discussed in [44] in which Runge-Kutta and Crank-Nicolson methods were applied. Other 
selective methodologies are [41,42] as well as $[42,43]$.

\subsection{General procedure}

Generally, if we bring the equations in the form $\dot{\mathbf{y}}=f(\mathbf{y}, t)$, then have to apply a method known from numerical analysis such as the Runge-Kutta of fourth order with variable time step [57]. Among others, an ideal function is ode 45 built-in in MATLAB $^{\circledR}$.

\subsection{Totality of dynamic Euler's equations}

The differential equations (15a) and (15b) for a system in which the body-fixed components $M_{1}$ and $M_{2}$ are expressed in terms of the Euler angles $(\theta, \psi)$, according to Eq. (16).

If the body-fixed angular velocity components $\left(\omega_{1}, \omega_{2}\right)$ could be found (note that $\omega_{3}$ is of given value), then Eq. (12) could be solved in $(\dot{\phi}, \dot{\theta}, \dot{\psi})$, say applying Cramer's rule, resulting in:

$$
\begin{aligned}
& \dot{\phi}=\frac{\omega_{1} \sin \psi+\omega_{2} \cos \psi}{\sin \theta} \\
& \dot{\theta}=\omega_{1} \cos \psi-\omega_{2} \sin \psi \\
& \dot{\psi}=\omega_{3}-\dot{\phi} \cos \theta
\end{aligned}
$$

Therefore, setting the five variables:

$$
y_{1}=\omega_{1}, y_{2}=\omega_{2}, y_{3}=\phi, y_{4}=\theta, y_{5}=\psi,
$$

the first-order system to be solved comprises the following five differential equations:

$$
\begin{aligned}
& \dot{y}_{1}=\frac{\left(I_{2}-I_{3}\right) \omega_{3}}{I_{1}} y_{2}+\frac{m g l \sin y_{4} \cos y_{5}}{I_{1}} \\
& \dot{y}_{2}=\frac{\left(I_{3}-I_{1}\right) \omega_{3}}{I_{2}} y_{1}-\frac{m g l \sin y_{4} \sin y_{5}}{I_{2}} \\
& \dot{y}_{3}=\frac{y_{1} \sin y_{5}+y_{2} \cos y_{5}}{\sin y_{4}} \\
& \dot{y}_{4}=y_{1} \cos y_{5}-y_{2} \sin y_{5} \\
& \dot{y}_{5}=\omega_{3}-\dot{y}_{3} \cos y_{4}
\end{aligned}
$$

\subsection{Based on space-fixed formulation}

Regarding the first Euler Equation, although it seems to be of the second order in $\phi$, it is finally of the first order [see, Eq. (38a)], thus we can set the first variable as $y_{1}=\phi$. Setting also $y_{2}=\theta$ and then solving in $\dot{\phi}$ Eq. (39b), which is equivalent to Eq. (39a), we receive the following ODE:

$$
\dot{y}_{1}=\frac{\left(c_{1}-I_{3} \omega_{3} \cos \theta\right)}{I_{1} \sin ^{2} \theta}=\frac{\left(c_{1}-I_{3} \omega_{3} \cos y_{2}\right)}{I_{1} \sin ^{2} y_{2}}
$$

In contrast, the second Euler equation is of the second order [see, Eq. (38b)] thus we need two variables, i.e. $y_{2}=\theta$ and $y_{3}=\dot{\theta}$. Practically, since $\omega_{3}$ is a constant, we do not need a new equation, thus the minimum necessary vector of unknowns to be involved in time integration (say Runge-Kutta) is $\mathbf{y}=\left[\begin{array}{lll}y_{1} & y_{2} & y_{3}\end{array}\right]^{T}=\left[\begin{array}{lll}\phi & \theta & \dot{\theta}\end{array}\right]^{T}$. The rest two ODEs will be:

$$
\dot{y}_{2}=y_{3}
$$

and

$$
\dot{y}_{3}=\frac{1}{I_{1}}\left(m g l \sin y_{2}+I_{1} \dot{y}_{1}^{2} \sin y_{2} \cos y_{2}-I_{3} \omega_{3} \dot{y}_{1} \sin y_{2}\right)
$$

Even if we wish to calculate the variation of the spin $\dot{\psi}$, after we have finished with the computation of the above-mentioned vector $\mathbf{y}$ in the desired time span, it is quite trivial to use the formula $\dot{\psi}=\omega_{3}-\dot{\phi} \cos \theta$ and compute it. But if someone wishes anyway to compute the spin angle $\psi$ itself, then one may include in the system the additional variable $y_{4}=\psi$. If these procedures are followed, an easy way to evaluate the accuracy of the numerical solution is to test the conservation of energy. On this issue, it was found that the default settings in the 'ode 45' MATLAB function (i.e. default tolerance of $10^{-6}$ ) lead to a numerical solution in which a minor decrease of the total energy is observed. This shortcoming is easily resolved by reducing the settings in active tolerance up to $10^{-14}$ at which the smoothest and most accurate numerical solution appears. In the latter case the number of time steps highly increases but the elapsed time is a few seconds only.

\subsection{Decoupled equation for the lean angle}

If we perform operations in Eq. (57c) we eventually obtain Eq. (44), which is computer programmed as follows. The primary variable is now chosen as 
$y_{1}=\theta$ with $y_{2}=\dot{\theta}$, so we could reduce our analysis to the system of two ODEs:

$$
\begin{gathered}
\dot{y}_{1}=y_{2} \\
\dot{y}_{2}=\frac{m g l \sin y_{1}}{I_{1}} \\
-\frac{\left(I_{3} \omega_{3}-c_{1} \cos y_{1}\right)\left(c_{1}-I_{3} \omega_{3} \cos y_{1}\right)}{I_{1}^{2} \sin ^{3} y_{1}}
\end{gathered}
$$

The system of Eq. $(58 \mathrm{a}, \mathrm{b})$ can be easily solved to numerically derive the functions $\theta(t)$ and $\dot{\theta}(t)$.

Moreover, one may proceed as follows. After the numerical solution of the lean angle $\theta(t)$ is completed, one may use Eq. (43) to determine $\dot{\phi}$ and then by integration in time $t$ to determine the Euler angle $\phi(t)$. Then, the third Euler equation:

$$
\dot{\psi}=\omega_{3}-\dot{\phi} \cos \theta
$$

can be optionally used only if we wish to determine the spin angle $\psi(t)$.

Of course, one may include all three equations, i.e. Eqs. (43), (44) and (59), in the same system of the form $\dot{\mathbf{y}}=f(\mathbf{y}, t)$.

\subsection{Based on energy conservation}

Theoretically, one equation in the system that describes the motion of the spinning top could be replaced by the energy conservation $[15,16]$. Within this context, substituting Eq. (43) into Eq. (42), and then solving in $\dot{\theta}$, one obtains:

$$
\dot{\theta}= \pm\left\{\frac{2}{I_{1}}\left(c_{2}-\frac{1}{2} I_{3} \omega_{3}^{2}-m g l \cos \theta\right)-\left[\frac{\left(c_{1}-I_{3} \omega_{3} \cos \theta\right)}{I_{1} \sin \theta}\right]^{2}\right\}^{1 / 2}
$$

While a lot of work has been devoted to implement elliptic integrals in Eq. (60) [4-7], under certain circumstances Eq. (60) can be also integrated in a numerical way. One difficulty is that the sign preserves its value only between two successive 'turning' points of the nutation provided the initial derivative is different than zero, In particular, when the initial condition is $\dot{\theta}_{0}=0$, neither of the RungeKutta or the Crank-Nicolson procedures is possible. But even if $\dot{\theta}_{0} \neq 0$ Eq. (60) is valid only until the next turning point at which $\dot{\theta}_{\text {turn }}=0$

\section{Application example}

As an application, we study a slow top previously used in literature [33,37]. In more detail, the geometric data were: $I_{1}=I_{2}=\frac{7}{8}, I_{3}=\frac{1}{4}$ and $l=\frac{\sqrt{3}}{2} m$, while the initial data where chosen to be $\phi_{0}=0, \dot{\phi}_{0}=0, \quad \theta_{0}=\pi / 16, \dot{\theta}_{0}=0, \quad$ and $\psi_{0}=\pi / 16, \dot{\psi}_{0}=1$. The mass of the top was taken $m$ $=1 \mathrm{~kg}$.

Using the ode45 MATLAB solution in conjunction with four variables (see above), after 248 steps of variable size, the results are shown in Fig. 4.

Figure 4: Euler angles (in radians) and distance (in meters) of the center of mass from the horizontal level.

The support forces are shown in Fig. 5.

Figure 5: Calculated support forces 
Figure 6: Calculated horizontal support force components

One may observe that the vertical support force $F_{z}$ may become up to 4.4 times larger than the dead weight (red line) and may also vanish or even take a small negative value ( -0.13 times the dead weight).

Finally, regarding the horizontal support forces, the maximum $F_{x}$ is about 84 percent of the dead weight, whereas maximum $F_{y}$ reaches about 2.6 times the dead weight, as shown in Fig. 6.

\section{Discussion}

It was shown that all the four formulations we presented are equivalent each other and lead to the same set of ordinary differential equations (ODE), which are called Euler dynamic equations. Although older researchers devoted many years to implement closed-form analytical expressions such as elliptic integrals, it was found that simple numerical analysis schemes (such as Runge-Kutta) are applicable and easily lead to nice graphs of the Euler angles versus time.

In all these approaches, the simplest ODE is of the first order with respect to the azimuthal angle $\phi$ and ensures the conservation of the angular momentum in the vertical space $z$-axis $\left(\dot{L}_{z}=0\right)$.

It is also remarkable that the second (Euler) ODE is of the second order with respect to the lean angle $\theta$, and is nothing else but Newton's second law in rotation with respect to either of the horizontal Cartesian axes $O x$ and $O y$. Clearly, the interchangeability of these two axes occurs because the moce ment vector $\mathbf{M}_{\text {space }}=m g l \sin \theta\left[\begin{array}{lll}\cos \phi & \sin \phi & 0\end{array}\right]^{T}$ always lies onto the horizontal plane $O x y$ and rotates according to the azimuthal angle $\phi(t)$. In more detail, the angular momentum is not preserved in the $x$ - and $y$ directions [see, Eq. (23a) and Eq. (23b)] but, closely related, their derivatives over time differ from zero according to Eqs. (24a) and (24b), being equal to the corresponding components of the external torque $\mathbf{M}_{\text {space }}$

In all the four formulations, the third Euler equation, i.e. (14c) of the symmetric spinning top (i.e., $I_{1}=I_{2}$ ) leads to the condition that the quantity $\omega_{3}=\dot{\phi} \cos \theta+\dot{\psi}$ is a constant (of course if it never reaches the ground). This means that not only it is possible that the spin $\dot{\psi}$ varies, but -under certain conditions- its direction may also be reversed [58].

Despite the complexity and the coupling between the three Euler angles $(\phi, \theta, \psi)$, it was made possible to decouple them and reveal an ordinary differential equation [Eq. (44)] which includes only the lean angle $\theta(t)$. Again, we recall that the lean (or inclination) angle is by definition related to the nutation.

As a check of the theory, we shall validate that Eq. (44) covers the so-called "regular" or "steady" or "smooth" precession as well, in which $\theta(t)=\pi / 2$ and no nutation appears. Actually, setting $\theta(t)=\theta_{0}=\pi / 2$ in Eq. (43), we receive $c_{1}=I_{1} \dot{\phi}_{0}$ and then substituting in Eq. (44) with $\ddot{\theta}=0$, one obtains $\dot{\phi}_{0}=(m g l) /\left(I_{3} \omega_{3}\right)$, which is the standard expression for the regular precession, which may be found in all elementary textbooks of physics $[15,19,20]$, mechanics $[16,17]$ and mathematics [53].

Of course, there are more other ways to obtain the Euler equations. For example, in the third formulation (section 5) the external moment was taken with respect to the fixed point $O$. But it is well known that, in the most general case in which the point $O$ is not fixed (see, for example [49] when it rotates along a specified circular path), the moments should be taken with respect to the center of mass $C$, instead. Within this context, the student may derive the same Euler equations, this time considering a different rotating system originated at the center of mass $C$.

\section{Conclusions}

Among the four ways which were presented with the aim to derive the governing ODEs for the symmetrical fixed spinning top, the one related to a 
non-spinning rotating system is probably the most convenient, but also the Lagrangian approach is straight forward. It was found that all of them lead to the same set of two governing equations, which in any case include the azimuthal and the inclination angles, while the spin is a secondary quantity.

The findings may be summarized as follows:

- Generally, the Euler equations cannot be integrated as they are, because the external moments are not known functions of the bodyfixed angular velocity components $\left(\omega_{1}, \omega_{2}, \omega_{3}\right)$.

- However, if the external moments equal to zero, of if they are known functions of the body-fixed angular velocity, the time integration in terms of $\omega_{1}, \omega_{2}$ and $\omega_{3}$ is possible.

- The well known Newton's second law in rotation, according to which the external moment equals to the derivative of the angular momentum in time, is not applicable to the body-fixed system as is.

- Of course, the Newton's second law in rotation is applicable to the space-fixed system.

- The two components $\left(L_{x}, L_{y}\right)$ of the angular momentum on the horizontal plane $\mathrm{O} x_{s} y_{s}$ lead to the same ODE.

- The space $z_{s}$-component $L_{z}$ of the angular momentum is an invariant quantity thus its derivative vanishes. The latter formulation is the second (Euler's) governing equation.

- The two abovementioned discrete ODEs are of the first and second order, respectively, expressed in only the variables $\phi$ and $\theta$, that is eventually they do not include the spin angle $\psi$.

- For each time $t$, the spin is easily determined by the relation in terms of the calculated azimuthal and lean angles. Usually, there is no practical need to calculate separately all the three components $\left(\omega_{1}, \omega_{2}, \omega_{3}\right)$, not even the spin. The latter can be calculated afterwards.

- Similarly, when implementing the Lagrange equations, the spin does not appear at all.

\section{Appendix A}

\section{Derivation of Euler equations of motion using transformation matrices}

In general, the relation between the co-ordinates of a vector $\mathbf{v}$ (tensor of rank one) in the body- $\left(\mathrm{O} x_{b} y_{b} z_{b}\right)$ and the space- $\left(\mathrm{O} x_{s} y_{s} z_{s}\right)$ system, is:

$$
\mathbf{v}_{\text {space }}=\mathbf{A}^{T} \cdot \mathbf{v}_{\text {body }},
$$

In our case of interest, $\mathbf{A}$ is the transformation matrix given by Eq. (9).

Considering the angular velocity in matrix form (which is the same as the matrix $\boldsymbol{\varepsilon}$ that is cited on a classical textbook [16, p. 170], eq. (4.69')):

$$
\mathbf{\Omega}_{b o d y}=\left(\begin{array}{ccc}
0 & -\omega_{3} & \omega_{2} \\
\omega_{3} & 0 & -\omega_{1} \\
-\omega_{2} & \omega_{1} & 0
\end{array}\right),
$$

it can be easily proved that $\dot{\mathbf{A}}=\boldsymbol{\Omega}_{b o d y}^{T} \cdot \mathbf{A}$, or equivalently

$$
(\dot{\mathbf{A}})^{T}=\mathbf{A}^{T} \cdot \mathbf{\Omega}_{b o d y}
$$

It is worth-mentioning that the definition of $\boldsymbol{\Omega}$ in (A-2) is so as it fulfills the identity: $\boldsymbol{\Omega} \cdot \mathbf{v}=\overrightarrow{\boldsymbol{\omega}} \times \overrightarrow{\mathbf{v}}$ (matrix product equals to the cross product).

The second Newton's law for rotation holds in only the space system and can be written as follows:

$$
\frac{d \mathbf{L}_{\text {space }}}{d t} \equiv \dot{\mathbf{L}}_{\text {space }}=\mathbf{M}_{\text {space }}
$$

Setting $\mathbf{v}=\mathbf{L}_{\text {space }}$ in (A-1) yields $\mathbf{L}_{\text {space }}=\mathbf{A}^{T} \cdot \mathbf{L}_{\text {body }}$ thus the product rule on the latter function is

$$
\dot{\mathbf{L}}_{\text {space }}=\left(\mathbf{A}^{T} \cdot \mathbf{L}_{\text {body }}\right)^{\cdot}=\mathbf{A}^{T} \dot{\mathbf{L}}_{\text {body }}+\left(\mathbf{A}^{T}\right)^{\cdot} \cdot \mathbf{L}_{\text {body }}
$$

Substituting (A-3) into (A-5) we obtain:

$$
\dot{\mathbf{L}}_{\text {space }}=\mathbf{A}^{T} \dot{\mathbf{L}}_{b o d y}+\mathbf{A}^{T} \cdot \mathbf{\Omega}_{b o d y} \cdot \mathbf{L}_{b o d y}
$$

Now, setting $\mathbf{v}=\mathbf{M}_{\text {space }}$ in (A-1) yields:

$$
\mathbf{M}_{\text {space }}=\mathbf{A}^{T} \cdot \mathbf{M}_{b o d y},
$$

Substituting (A-6) and (A-7) into (A-4), and then clearing the common factor $\mathbf{A}^{T}$ (otherwise left-multiplying by the matrix $\mathbf{A}$ which fulfils the relation $\mathbf{A} \mathbf{A}^{T}=\mathbf{I}=$ identity matrix), we obtain

$$
\dot{\mathbf{L}}_{b o d y}+\boldsymbol{\Omega}_{b o d y} \cdot \mathbf{L}_{b o d y}=\mathbf{M}_{b o d y}
$$

Considering that

$$
\dot{\mathbf{L}}_{b o d y}=\left[\begin{array}{lll}
I_{1} \dot{\omega}_{1} & I_{2} \dot{\omega}_{2} & I_{3} \dot{\omega}_{3}
\end{array}\right]^{T}
$$

and

$$
\mathbf{M}_{b o d y}=\left[\begin{array}{lll}
M_{1} & M_{2} & M_{3}
\end{array}\right]^{T},
$$


Equation (A-8) becomes

$$
\left[\begin{array}{c}
I_{1} \dot{\omega}_{1} \\
I_{2} \dot{\omega}_{2} \\
I_{3} \dot{\omega}_{3}
\end{array}\right]+\left(\begin{array}{ccc}
0 & -\omega_{3} & \omega_{2} \\
\omega_{3} & 0 & -\omega_{1} \\
-\omega_{2} & \omega_{1} & 0
\end{array}\right) \cdot\left[\begin{array}{c}
I_{1} \omega_{1} \\
I_{2} \omega_{2} \\
I_{3} \omega_{3}
\end{array}\right]=\left[\begin{array}{c}
M_{1} \\
M_{2} \\
M_{3}
\end{array}\right]
$$

One may observe that Eq. (A-11) is identical with Eq. $(14 \mathrm{a}, \mathrm{b}, \mathrm{c})$, and this completes the proof.

\section{Appendix B}

\section{Moment induced by the weight}

Consider the situation shown in Fig. 1, which is repeated in Fig. 7. The moment vector $\boldsymbol{\tau}_{\text {ext }}$ induced by the weight $\overrightarrow{\mathbf{W}}=-m \overrightarrow{\mathbf{g}}$ with respect to the fixed point $O$ is given by:

$$
\boldsymbol{\tau}_{e x t}=\overrightarrow{\mathbf{r}} \times(-m \overrightarrow{\mathbf{g}}),
$$

Since the position vector $\overrightarrow{\mathbf{r}}$ is $\overrightarrow{O C}$, the moment vector will lie along the line of nodes $O N$, directed from $O$ to $N$. The magnitude of the moment will be equal to $m g \rho=m g l \sin \theta$. Also, the vector $\overrightarrow{\boldsymbol{\tau}}_{\text {ext }}$ splits in two Cartesian components:

$$
\begin{aligned}
& M_{x}=-(m g l \sin \theta) \sin \phi_{P} \\
& M_{y}=+(m g l \sin \theta) \cos \phi_{P}
\end{aligned}
$$

Due to Eq. (43), Eq. (B-2) becomes:

$$
\begin{aligned}
& M_{x}=(m g l \sin \theta) \cos \phi \\
& M_{y}=(m g l \sin \theta) \sin \phi
\end{aligned}
$$

In vector form, Eq. (B-3) is rewritten as

$$
\left[\begin{array}{l}
M_{x} \\
M_{y}
\end{array}\right]=m g l \sin \theta\left[\begin{array}{c}
\cos \phi \\
\sin \phi
\end{array}\right],
$$

where $\phi$ is the classical Euler angle $\phi=\square\left(O x_{s}, O N\right)$, and not the polar angle $\phi_{P}=\square\left(O x_{s}, O C^{\prime}\right)$. Obviously, $M_{z}=0$.

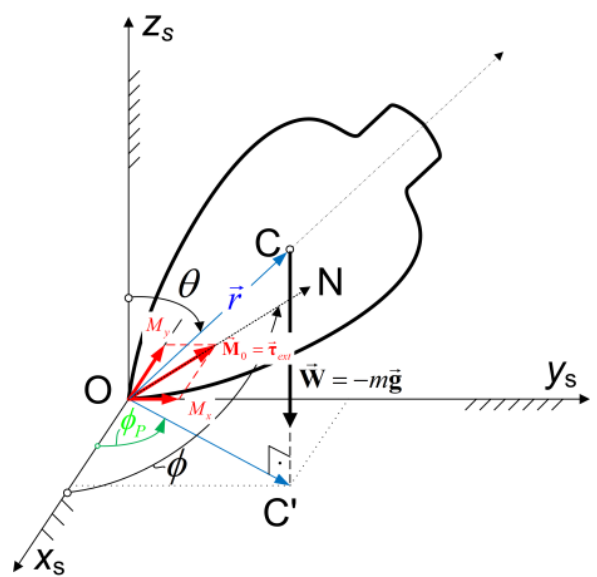

Figure 7: External moment

\section{References:}

[1] Lawerance A., Modern Inertial Technologies: Navigation, Guidance and Control. Springer, New York (1992)

[2] Rimrott F.P.J, Introductory Attitude Dynamics. Springer, New York (1989)

[3] Townsend, N., Shenoi, A.: A gyroscopic wave energy recovery system for marine vessels, IEEE $J$. Ocean. Eng. 37(2), 271-280 (2012)

[4] Passaro VMN, Cuccovillo A, Vaiani L, Carlo M, Campanella CE. Gyroscope Technology and Applications: A Review in the Industrial Perspective. Sensors (Basel). 2017;17(10):2284. Published 2017 Oct 7. doi:10.3390/s17102284

[5] Lagrange J. L., Mécanique Analytique, Courcier, Paris (1811)

[6] Klein F., and Sommerfeld A., The Theory of the Top, Volume 1: Introduction to the Kinematics and Kinetics of the Top, Birkhäuser, Boston (2008); translated by R J Nagen and G Sandri (Originally published 1897 by Teubner)

[7] Klein F., and Sommerfeld A., The Theory of the Top, Volume 2: Development of the Theory in the Case of the Heavy Symmetric Top, Birkhäuser, Boston (2010); translated by R J Nagen and G Sandri (Originally published 1898 by Teubner)

[8] Klein F., The Mathematical Theory of the Top, Charles Scribner Sons, New York (1987). Republished in 2004 (Dover, Mineola).

[9] Appel'rot G.G., The problem of motion of a rigid body about a fixed point, Uchenye Zap. Mosk.Univ. Otdel.Fiz. Mat. Nauk, 11, 1-112 (1894)

[10] Crabtree H., An Elementary Treatment of the Theory of Spinning Tops and Gyroscopic Motion, Longmans-Green-and Co, New York (1909)

[11] John Perry, Spinning Tops, E. S. Gorham, New York (1910). Also: "Spinning Tops and Gyroscopic Motion," Dover Publications, New York (1957)

[12] Gould D.W., The Top: Universal Toy Enduring Pastime, Bailey Brothers and Swinfen Ltd, Folkestone, UK (1975) 
[13] Whittaker E.T., A Treatise on the Analytical Dynamics of Particles and Rigid Bodies, Dover, New York (1944)

[14] Zaroodny S.J., An Elementary Introduction to Elliptic Functions Based on the Theory of Nutation, The American Mathematical Monthly, 68(7), 593616 (1961)

[15] French A.P., Newtonian Mechanics: The M.I.T. Introductory Physics Series, W. W. Norton \& Company Inc., New York (1971)

[16] Goldstein H., Poole C., and Safko J., Classical Mechanics, 3rd edition, Pearson Education Inc. (Addison Wesley), San Francisco (2002)

[17] Targ S., Theoretical Mechanics: A Short Course, Mir Publishers, Moscow, 3rd edition (1976)

[18] Arnold V.I., Mathematical Methods of Classical Mechanics, vol. 60, Springer-Verlag, New YorkHeidelberg-Berlin (1984)

[19] Halliday D., Resnick R., and Walker J., Fundamental of Physics, 9th edition, John Wiley \& Sons, NJ (2011), pp. 294-295.

[20] Young H.D., and Freedman R.A., University Physics, 12th edition, Pearson Addison-Wesley, San Francisco (2008), pp. 337-340.

[21] http://www.gyroscopes.org/index.asp

[22] S.Kowalevski, Sur le problème de la rotation d'un corps solide autour d'un point fixe, Acta Mathematica, 12 (1889).

[23] S.Kowalevski, Sur une propriété du système d'équations différentielles qui définit la rotation d'un corps solide autour d'un point fixe, Acta Mathematica, 14 (1889)

[24] R. Cooke, The mathematics of Sonya Kovalevskaya, Springer-Verlag, New York (1984)

[25] Goriely A., Nizette M., Kovalevskaya rods and Kovalevskaya waves, Regular and Chaotic Dynamics, 5(1), 95-106 (2000).

[26] Michèle Audin, Spinning Tops: A Course on Integrable Systems, Studies in Advanced Mathematics, Vol. 51, Cambridge University Press, Cambridge (1996)

[27] Golubev V. V., Lectures on integration of the equations of motion of a rigid body about a fixed point, Gostenhizdat, Moscow, 1953 [in Russian]; English translation: Philadelphia: Coronet Books (1953)

[28] Cushman R.H., and Bates L.M., Global Aspects of Classical Integrable Systems, Birkhäuser Verlag, Basel, 1997 (Chapter III: The Euler Top, pp. 83146).

[29] Saul Gorn, The Automatic Analysis and Control of Computing Errors, Journal of the Society for Industrial and Applied Mathematics, SIAM, 2(2), 69-81 (1954)

[30] McGill D.J. and Long L. S., III, Stability regions for an unsymmetrical rigid body spinning about an axis through a fixed point, Acta Mechanica 22, 91-112 (1975)

[31] Simo J.C. and Wong K.K., "Unconditionally stable algorithms for rigid body dynamics that exactly preserve energy and momentum," Int. J. Numer. Meth. Engng 31 (1991), 19-52.

[32] Simo J.C., Tarnow N. and Wong K.K., Exact energy-momentum conserving algorithms and symplectic schemes for nonlinear dynamics, Comp. Meth. Appl. Mech. Engng 100, 63-116 (1992)

[33] Ratiu T.and van Moerbeke P., The Lagrange rigid body notion, Ann. Inst. Fourier, Grenoble, 32 (1), 211-234 (1982)

[34] Romano M., Exact analytic solutions for the rotation of an axially symmetric rigid body subjected to a constant torque, Celest. Mech. Dyn. Astr. 101, 375390 (2008)

[35] Jackiewicz Z., Marthinsen A. and Owren B., Construction of Runge-Kutta methods of CrouchGrossman type of high order, Advances in Computational Mathematics 13, 405-415 (2000)

[36] Engø K. and Marthinsen A., A Note on the Numerical Solution of the Heavy Top Equations, Multibody System Dynamics 5, 387-397 (2001)

[37] Bou-Rabee N.M., Marsden J.E. and Romero L.A., Tippe Top Inversion as a Dissipation-Induced Instability, SIAM J. Applied Dynamical Systems, 3(3), 352-377 (2004)

[38] Kozlov R., High-order conservative discretizations for some cases of the rigid body motion, Physics Letters A, 373, 23-29 (2008)

[39] Owren B. and Marthiinsen A., Runge-Kutta methods adapted to manifolds and based on rigid frames, BIT, 39(1), 116-142 (1999) (particularly pp. 137-140)

[40] A.C. Or, The dynamics of a Tippe Top, SIAM Journal of Applied Mathematics, 54(3), 597-609 (1994)

[41] van Zon R., Schofield J., Numerical implementation of the exact dynamics of free rigid bodies, Journal of Computational Physics 225, 145-164 (2007)

[42] Tsiotras P., and Longuski J.M., Analytic Solution of Euler's Equations of Motion for an Asymmetric Rigid Body, ASME Journal of Applied Mechanics 63(1), 149-155 (1996)

[43] Belokolos E.D., Bobenko A.I., Enol'skii V.Z., Its A.R., and Matveev V.B., Algebro-Geometric Approach to Nonlinear Integrable Equations, Speinger-Verlag, Berlin-Heidelberg, 1994.

[44] Provatidis C.G., Revisiting the Spinning Top, International Journal of Materials and Mechanical Engineering, Vol. 1, No. 4 (July 2012), pp. 71-88 (Print ISSN: 2162-0695, Online ISSN: 2164-280X. The Journal ceased. If not found, download from: https://www.academia.edu/27516500/Revisiting_the_Spinning_Top.

[45] Cross R., A spinning top for physics experiments, Phys. Educ. 54 (2019) 055028 (4pp).

[46] Tanrıverdi V., Dissipative motion of a spinning heavy symmetric top, Eur. J. Phys. 41 (2020) 055001 (12pp)

[47] Usubamatov R., Theory of Gyroscopic Effects for Rotating Objects, Springer, Singapore, 2020.

[48] Thompson J.M.T., Instabilities of Elastic and Spinning Systems: Concepts and Phenomena, 
International Journal of Bifurcation and Chaos, Vol. 27, No. 9 (2017) 1730029 (26 pages)

[49] Provatidis C.G., Forced precession in a spinning wheel supported on a rotating pivot, Mech. Res. Com. 52, 46- 51 (2013)

[50] Sheheitli H., Touma J.R., On the dynamics of a spinning top under the influence of rotation: Resonant relative equilibrium states, Commun Nonlinear Sci Numer Simulat 59 (2018) 424-436

[51] Provatidis C.G., Forced precession of a gyroscope with application to the Laithwaite's engine, European Journal of Physics, 42035003 (2021).

[52] M.V. Berry and P. Shukla, Slow manifold and Hannay angle in the spinning top, Eur. J. Phys., 32, 115-127 (2011)

[53] Hay G.E., Vector and Tensor Analysis, Dover Publications, New York (1953), Chapter 3.

[54] Provatidis C.G., Free fall of a symmetrical gyroscope in vacuum, European Journal of Physics, (under revision).

[55] Panayotounakos D.E., Theocaris P.S., On the Decoupling and the Solutions of the Euler Dynamic Equations Governing the Motion of a Gyroscope, ZAMM, 70(11), 1990, pp. 489-500.

[56] Panayotounakos D.E., Rizou I., Theotokoglou E.E., A new mathematical construction of the general nonlinear ODEs of motion in rigid body dynamics (Euler's equations), Applied Mathematics and Computation, 217 (21), 1 July 2011, pp. 8534-8542.

[57] Press W.H., Teukolsky S.A., Vetterling W.T., Flannery B.P. (2007), "Section 17.1 Runge-Kutta Method", Numerical Recipes: The Art of Scientific Computing (3rd ed.), Cambridge University Press.

[58] Tanriverdi V., Can a gyroscope reverse its spin direction? 2019 Eur. J. Phys. 40065004

\section{Creative Commons Attribution}

\section{License 4.0 (Attribution 4.0 International, CC BY 4.0)}

This article is published under the terms of the Creative Commons Attribution License 4.0 https://creativecommons.org/licenses/by/4.0/deed.en US 